\title{
Adipostatins A-D from Streptomyces sp. 4875 inhibiting Brugia malayi asparaginyl-tRNA synthetase and killing adult Brugia malayi parasites
}

\author{
Mostafa E Rateb ${ }^{1}$, Dong Yang ${ }^{1}$, Sanja Vodanovic-Jankovic ${ }^{2}$, Zhiguo Yu${ }^{1}$, Michael A Kron ${ }^{2}$ and Ben Shen ${ }^{1,3,4}$ \\ The Journal of Antibiotics (2015) 68, 540-542; doi:10.1038/ja.2015.22; published online 4 March 2015
}

Lymphatic filariasis (LF), also known as elephantiasis, is one of the World Health Organization's (WHO's) top 10 neglected tropical diseases. ${ }^{1}$ LF is caused primarily by two related parasites, Brugia malayi and Wuchereria bancrofti, and represents a worldwide health crisis with over 200 million people infected and another $20 \%$ of the global population at risk for infection. ${ }^{1,2}$ Aminoacyl-tRNA synthetases (AARSs) were the first filarial targets for antiparasite drug discovery embraced by the WHO and are generally regarded as excellent therapeutic targets because AARSs have a key role in protein synthesis. ${ }^{3}$ Among the AARSs, in particular, asparaginyl-tRNA synthetase (AsnRS) in B. malayi is considered an excellent antifilarial target because (i) it is highly expressed in both sexes, adults and larvae of B. malayi; (ii) it is well characterized biochemically and structurally in B. malayi; (iii) it shows significant structural differences in comparison with human AARSs; and (iv) a high-throughput screening (HTS) platform with recombinant $B$. malayi AsnRS (BmAsnRS) for inhibitors has been developed. ${ }^{2,4-6}$

Natural products remain a valuable source of new drug leads and have demonstrated almost limitless potential in showcasing new molecular scaffolds with clinically relevant biological activities. ${ }^{7}$ In an effort to discover drug leads targeting BmAsnRS, we recently completed a HTS campaign of $\sim 73000$ extracts from a collection of 36720 microbial strains. Of the extracts screened, 177 active strains were identified. Subsequent bioassay-guided dereplication of two of the active strains resulted in the discovery of two natural product scaffolds as promising antifilarial drug leads, represented by tirandamycin B (TAM B) from Streptomyces sp. $17944^{8}$ and the depsipeptide WS9326D from Streptomyces sp. 9078. ${ }^{9}$ TAM B and WS9326D kill the adult $B$. malayi parasites at low nanomolar concentrations yet do not exhibit significant general cytotoxicity to human hepatic cells. ${ }^{8,9}$

Here we now report the bioassay-guided dereplication of another active strain, Streptomyces sp. 4875, leading to the discovery of four alkylresorcinols, named adipostatin A (1), adipostatin B (2), adipostatin C (3) and adipostatin D (4; Figure 1). All four adipostatins inhibit BmAsnRS and efficiently kill the adult B. malayi parasite. The adipostatins, which have not been recognized previously for use in the treatment of LF, represent another lead scaffold for antifilarial drug discovery. ${ }^{8,9}$

Streptomyces sp. 4875 was identified as a Streptomyces species on the basis of its 16S ribosomal RNA (Supplementary Figure S1). A twostage fermentation of Streptomyces sp. 4875 was performed as reported before (Supplementary Information). ${ }^{8,9}$ The production culture (81) was extracted with 3\% Amberlite XAD-16 (Sigma-Aldrich, St Louis, MO, USA) resin to afford the crude extract. The crude extract was subjected to multiple steps of $\mathrm{SiO}_{2}$, Sephadex LH-20 and preparative C-18 chromatography. Dereplication and natural product isolation were guided by bioassay for inhibitory activity against the recombinant $B m A s n R S$, affording pure $\mathbf{1}(3 \mathrm{mg}), 2$ (4.2 mg), 3 (7 mg) and $4(5 \mathrm{mg})$ as white powders (Supplementary Information). The ${ }^{1} \mathrm{H}$ and ${ }^{13} \mathrm{C}$ NMR spectra of $\mathbf{1 - 4}$ were obtained in $\mathrm{CD}_{3} \mathrm{OD}$ (Table 1 and Supplementary Figures S2-S15).

Analysis of the HRESIMS data, the ${ }^{1} \mathrm{H}$ and ${ }^{13} \mathrm{C}$ NMR spectra and the 2D NMR data (Supplementary Figures S2-S11) of 1 and 2 suggested them to be adipostatin $\mathrm{A}$ and $\mathrm{B}$, respectively, two alkylresorcinol regioisomers that have been previously isolated from Streptomyces cyaneus $2299-S V 1^{10}$ and were patented as potent glyceryl3-phosphate dehydrogenase inhibitors. ${ }^{11}$ The identity of $\mathbf{1}$ and $\mathbf{2}$ was subsequently confirmed by NMR analysis (Supplementary Figures S2-S11), as well as comparison with the spectroscopic data reported previously (Table 1). ${ }^{10}$ HRESIMS analysis of $\mathbf{3}$ and $\mathbf{4}$ yielded the $[\mathrm{M}+\mathrm{H}]^{+}$ions at $m / z 335.2946$ and $m / z$ 335.2944, respectively, indicative that 3 and 4 were also regioisomers with the same molecular formula of $\mathrm{C}_{22} \mathrm{H}_{38} \mathrm{O}_{2}$ (calculated $[\mathrm{M}+\mathrm{H}]^{+}$ion for $\mathrm{C}_{22} \mathrm{H}_{38} \mathrm{O}_{2}$ at $\mathrm{m} / 2$ 335.2944) but differed from 1 and $2\left(\mathrm{C}_{21} \mathrm{H}_{36} \mathrm{O}_{2}\right)$ by an additional $\mathrm{CH}_{2}$ unit. On the basis of the ${ }^{1} \mathrm{H}$ and ${ }^{13} \mathrm{C}$ NMR data of 3 and 4 (Supplementary Figures S12-S15), as well as in comparison with those of 1 and 2 (Supplementary Figures S2-S11), 3 and 4 were established as adipostatins C (3) and D (4) (Figure 1). Although 3 and

${ }^{1}$ Department of Chemistry, The Scripps Research Institute, Jupiter, FL, USA; ${ }^{2}$ Biotechnology and Bioengineering Center and Department of Medicine, Medical College of Wisconsin, Milwaukee, WI, USA; ${ }^{3}$ Department of Molecular Therapeutics, The Scripps Research Institute, Jupiter, FL, USA and ${ }^{4}$ Natural Products Library Initiative, The Scripps Research Institute, Jupiter, FL, USA

Correspondence: Professor B Shen, Department of Chemistry, The Scripps Research Institute, 130 Scripps Way, \#3A1, Jupiter, FL 33458, USA

E-mail: shenb@scripps.edu

Received 25 November 2014; revised 26 January 2015; accepted 30 January 2015; published online 4 March 2015 
4 had been reported previously in the patent literature, ${ }^{11}$ their spectral data were not available. The ${ }^{1} \mathrm{H}$ and ${ }^{13} \mathrm{C}$ NMR data for $\mathbf{3}$ and $\mathbf{4}$ are therefore reported here, in comparison with $\mathbf{1}$ and $\mathbf{2}$ reported previously $^{10}$ (Table 1).

Each of the four purified adipostatins was re-evaluated for their inhibitory activity against BmAsnRS, using the recently reported pretransfer editing assay. ${ }^{6,8,9}$ Inhibition against BmAsnRS was observed for all the four adipostatins with apparent $\mathrm{IC}_{50} \mathrm{~s}$ estimated to be $15 \mu \mathrm{M}$ (for 1), $15 \mu \mathrm{M}$ (for 2), $15 \mu \mathrm{M}$ (for 3) and $30 \mu \mathrm{M}$ (for 4) (Supplementary Figure S16). We next tested the four adipostatins for their ability to kill adult $B$. malayi worms in vitro following the published procedure. ${ }^{8,9}$ Live adult $B$. malayi worms were maintained in six-well plates (three worms per well), and each of the four adipostatins, varying from $100 \mathrm{~nm}$ to $100 \mu \mathrm{M}$, was added to selected

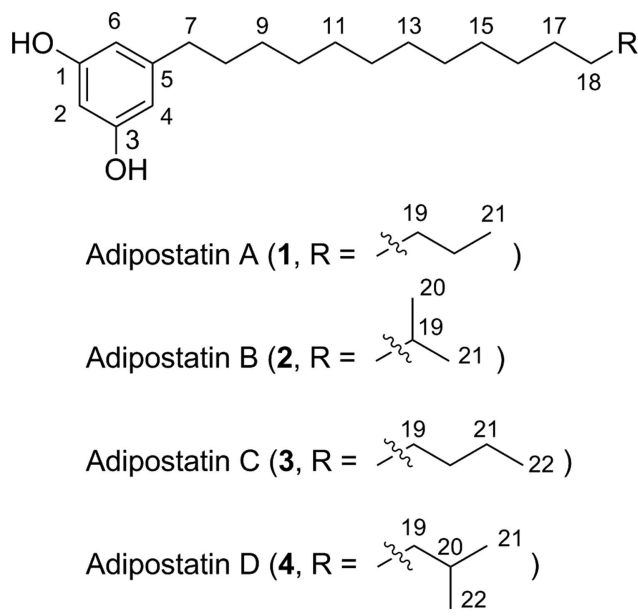

Figure 1 Structures of adipostatins A-D (1-4) from Streptomyces sp. 4875. wells to test their parasite-killing activity, with TAM B as positive controls (Supplementary Figures S17-S20). At $100 \mu \mathrm{M}$ concentration, 2 and 3 kill all the adult B. malayi worms rapidly, that is, within $24 \mathrm{~h}$, whereas at that concentration, 1 and 4 require $48 \mathrm{~h}$ for complete killing, with 3 and 1 efficiently kill the adult worms within 8 days and 9 days, respectively, at concentrations as low as $100 \mathrm{~nm}$ (Table 2). Worm death was unambiguously differentiated from simple paralysis by the modified MTT assay. ${ }^{8,9}$ Although 2 and 3 seemed to be more potent than 1 and 4 at higher concentrations (for example, at $100 \mu \mathrm{M}$ or $50 \mu \mathrm{M}), 1$ killed the worms sooner than 2 and 3 at lower concentrations (for example, at $1 \mu \mathrm{M}$ ). Finally, the four adipostatins were evaluated for general cytotoxicity by using human HepG2 cells. Cytotoxicity was defined as $>50 \%$ cell death at $24 \mathrm{~h}$ as measured by the MTT assay for mitochondrial activity, ${ }^{12}$ and all four adipostatins were found to be nontoxic at concentration as high as $100 \mu \mathrm{M}$ (Supplementary Figure S21). This finding, in combination with the ability of 1-4 to selectively inhibit the BmAsnRS and efficiently kill the adult $B$. malayi worms, constitutes the basis for continued chemical and biological efforts to understand the mode of action and the structure-activity relationship of the alkylresorcinols, as exemplified by the adipostatins, as a new lead scaffold for antifilarial drug discovery.

Table 2 Complete killing time of adult $B$. malayi worms by adipostatins A-D (1-4) at varying concentrations ${ }^{a}$

\begin{tabular}{lcccccc}
\hline & $100 \mu \mathrm{M}$ & $50 \mu \mathrm{M}$ & $25 \mu \mathrm{M}$ & $10 \mu \mathrm{M}$ & $1 \mu \mathrm{M}$ & $100 \mathrm{~nm}$ \\
\hline Adipostatin A (1) & 2 & 5 & 5 & 5 & 6 & 9 \\
Adipostatin B (2) & 1 & 3 & 8 & 8 & 9 & $-{ }^{\mathrm{b}}$ \\
Adipostatin C (3) & 1 & 2 & 3 & 4 & 8 & 8 \\
Adipostatin D (4) & 2 & 5 & 7 & 8 & 9 & $-^{\mathrm{b}}$ \\
\hline
\end{tabular}

aUnit of killing time: day.

${ }^{5}$ At least one of the three $B$. malayi worms in each of the wells was still alive after 10 days of treatment.

Table $1{ }^{1} \mathrm{H}(700 \mathrm{MHz})$ and ${ }^{13} \mathrm{C}(175 \mathrm{MHz})$ NMR data of adipostatins A-D (1-4) in $\mathrm{CD}_{3} \mathrm{OD}^{\mathrm{a}}$

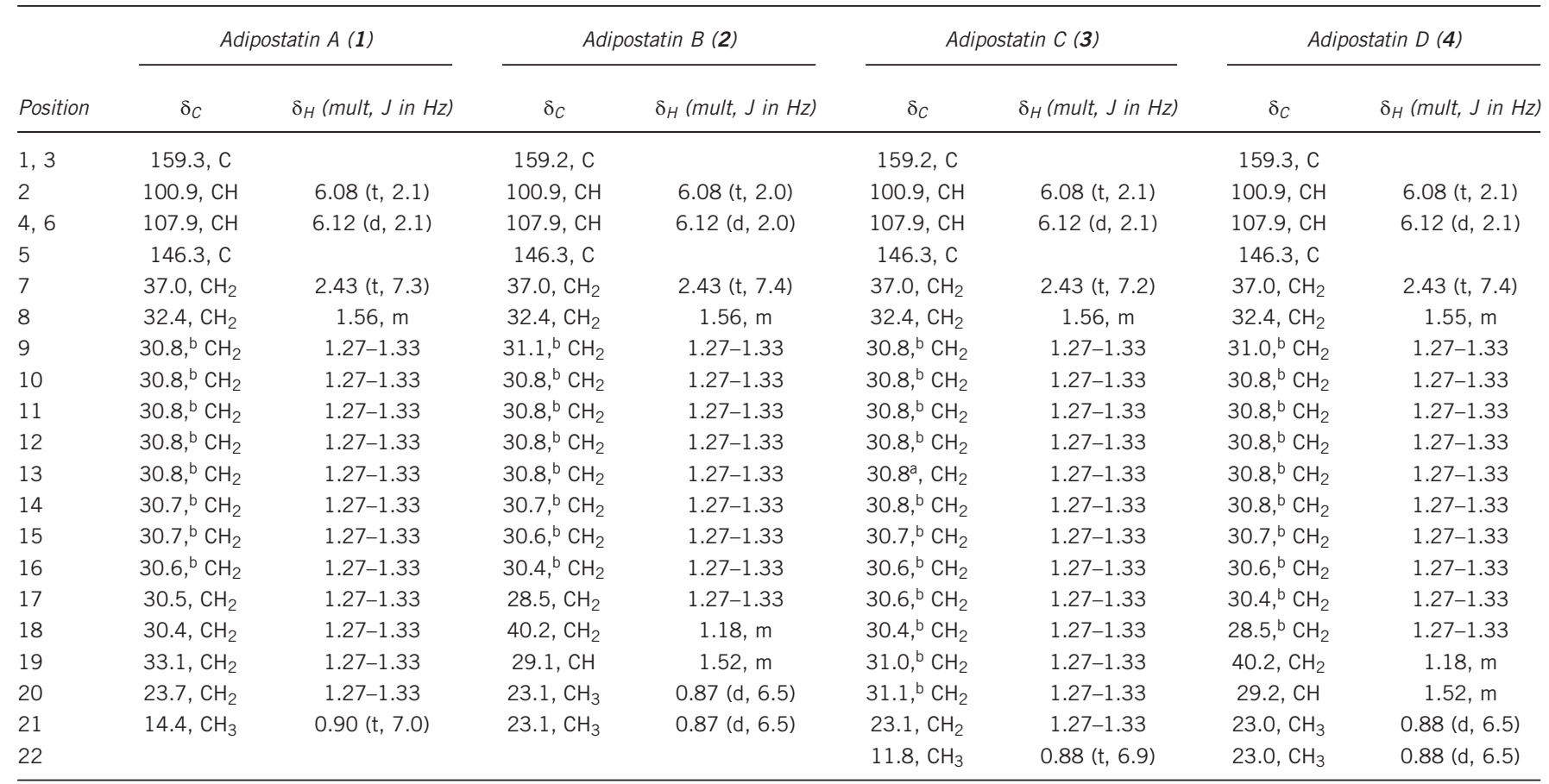

The ${ }^{1} \mathrm{H}$ and ${ }^{13} \mathrm{C}$ NMR data for $\mathbf{1}$ and $\mathbf{2}$, reported previously in $\mathrm{CDCl}_{3},{ }^{10}$ are included here in $\mathrm{CD}_{3} \mathrm{OD}$ for comparison with those of $\mathbf{3}$ and $\mathbf{4}$ also recorded in $\mathrm{CD}_{3} \mathrm{OD}$.

'Interchangeable. 
In summary, $\mathbf{1}$ and $\mathbf{2}$ were first isolated as glyceryl-3-phosphate dehydrogenase inhibitors from S. cyaneus 2299-SV1 two decades ago. ${ }^{10}$ Guided by the innovative HTS targeting BmAsnRS, we now report that $\mathbf{1}$ and $\mathbf{2}$, together with two additional congeners, 3 and $\mathbf{4}$, are novel $B m$ AsnRS inhibitors that efficiently kill the adult $B$. malayi parasites, but do not exhibit significant general cytotoxicity to human hepatic cells. The adipostatins therefore represent another natural product lead scaffold that could be exploited to combat the global health crisis of LF. These findings, together with our early reports of TAM B and WS9326D as promising antifilarial drug leads, ${ }^{8,9}$ underscore the great promise of our strategy in screening microbial natural products as BmAsnRS inhibitors for antifilarial drug discovery. The microbial origin of the natural product leads, thereby their availability by scale-up fermentation, should greatly facilitate the follow-up mechanistic and preclinical studies needed to advance the most promising leads into clinical drugs.

\section{ACKNOWLEDGEMENTS}

We thank the Filariasis Research Reagent Resources, Division of Microbiology and Infectious Disease, NIAID, NIH for providing adult B. malayi. This work was supported in part by NIH grants AI053877 (MAK) and AI105472 (BS and MAK).
1 Dzenowagis, J. Lymphatic Filariasis: Reasons for Hope (World Health Organization, Geneva, Switzerland, 1997)

2 Kron, M., Marquard, K., Hartlein, M., Price, S. \& Lederman, R. An immunodominant antigen of Brugia malayi is an asparaginyl-transfer-RNA synthetase. FEBS Lett. 374 122-124 (1995)

3 Kron, M., Yousin, F. \& Ramirez, B. Capacity building in anthelmintic drug discovery. Expert Opin. Drug Discov. 2, S1-S8 (2007).

4 Nilsen, T. W. et al. Cloning and characterization of a potentially protective antigen in lymphatic filariasis. Proc. Natl Acad. Sci. USA 85, 3604-3607 (1988).

5 Crepin, T. et al. A hybrid structural model of the complete Brugia malayi cytoplasmic asparaginyl-tRNA synthetase. J. Mol. Biol. 405, 1056-1069 (2011).

6 Danel, F. et al. Asparaginyl-tRNA synthetase pre-transfer editing assay. Curr. Drug Discov. Technol. 8, 66-75 (2011).

7 Newman, D. J. \& Cragg, G. M. Natural products as sources of new drugs over the 30 years from 1981 to 2010. J. Nat. Prod. 75, 311-335 (2012).

$8 \mathrm{Yu}, \mathrm{Z}$. et al. Tirandamycins from Streptomyces sp. 17944 inhibiting the parasite Brugia malayi asparagine tRNA synthetase. Org. Lett. 13, 2034-2037 (2011).

9 Yu, Z., Vodanovic-Jankovic, S., Kron, M. \& Shen, B. New WS9326A congeners from Streptomyces sp. 9078 inhibiting Brugia malayi asparaginyl-tRNA synthetase. Org. Lett. 14, 4946-4949 (2012).

10 Tsuge, N., Mizokami, M., Imai, S., Shimazu, A. \& Seto, H. Adipostatins A and B, new inhibitors of glycerol-3-phosphate dehydrogenase. J. Antibiot. 45, 886-891 (1992).

11 Seto, H., Shimazu, A., Imai, S., Tsuge, N. \& Mizokami, M. Alkylresorcinols as glycerophosphate dehydrogenase inhibitors. JP06100440 (1994).

12 Comley, J. C. W., Res, M. J., Turner, C. H. \& Jenkins, D. C. Colorimetric quantitation of filarial viability. Int. J. Parasitol. 19, 77-83 (1989).

Supplementary Information accompanies the paper on The Journal of Antibiotics website (http://www.nature.com/ja) 